Intersections

Canadian Journal of Music

Revue canadienne de musique
Intersections

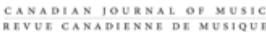

\title{
L'expérience musicale est une expérience sociale : de l'intellectualisation de la musique électronique à Lima (Pérou)
}

\section{Raúl Matta}

Volume 30, numéro 2, 2010

URI : https://id.erudit.org/iderudit/1006378ar

DOI : https://doi.org/10.7202/1006378ar

Aller au sommaire du numéro

Éditeur(s)

Canadian University Music Society / Société de musique des universités canadiennes

ISSN

1911-0146 (imprimé)

1918-512X (numérique)

Découvrir la revue

Citer cet article

Matta, R. (2010). L'expérience musicale est une expérience sociale : de l'intellectualisation de la musique électronique à Lima (Pérou). Intersections, 30(2), 61-74. https://doi.org/10.7202/1006378ar
Résumé de l'article

La musique ne saurait être analysée per se, car elle est enracinée dans les contextes sociaux dans lesquels elle se produit et se développe. En analysant les caractéristiques de l'espace social de diffusion de la musique électronique dans la ville de Lima, nous serons en mesure de comprendre la façon dont ce genre musical « mineur » est aujourd'hui devenu un vecteur de distinction sociale et d'élitisme.
All Rights Reserved (C Canadian University Music Society / Société de musique des universités canadiennes, 2011
Ce document est protégé par la loi sur le droit d'auteur. L'utilisation des services d'Érudit (y compris la reproduction) est assujettie à sa politique d'utilisation que vous pouvez consulter en ligne.

https://apropos.erudit.org/fr/usagers/politique-dutilisation/ 


\title{
L'EXPÉRIENCE MUSICALE EST UNE EXPÉRIENCE SOCIALE : DE L'INTELLECTUALISATION DE LA MUSIQUE ÉLECTRONIQUE À LIMA (PÉROU)
}

\author{
Raúl Matta
}

La sociologie de la consommation culturelle a essentiellement considéré les rapports à la culture sous l'angle des inégalités, en produisant des modèles de stratification sociale des goûts culturels qui établissent une correspondance entre milieux sociaux d'appartenance et préférences affichées ${ }^{1}$. En France, si des recherches plus récentes ont démontré que les rapports de domination liés au statut social s'expriment de moins en moins fortement dans les pratiques culturelles, elles n'ont pas pour autant réussi à invalider leur pouvoir explicatif (Donnat 2003, Coulangeon 2003).

En prenant comme exemple la musique électronique de danse à Lima, nous verrons que ces rapports n'ont rien perdu de leur efficacité lorsqu'il s'agit d'établir une hiérarchie au sein d'un genre musical ou au sein d'une scène culturelle. D’ailleurs, dans un espace social bien délimité et de taille plutôt réduite, les stratégies des groupes cherchant à asseoir leur hégémonie deviennent plus fines et parfois difficilement objectivables. Nous entendrons par musique électronique de danse une musique construite dans son intégralité par des machines, privilégiant les effets kinesthésiques dans un contexte festif, associée à l'image de son exécutant (le DJ), soucieuse de préserver une certaine indépendance par rapport aux grands groupes de l'industrie musicale (majors) et se rapprochant d'une attitude underground qui façonne ses propres innovations ${ }^{2}$.

1 Les modèles les plus représentatifs demeurent celui de Pierre Bourdieu (1979) et celui de Richard Peterson (1992). Bourdieu emploi le concept de légitimité culturelle pour montrer qu'il existe, dans l'espace social, une lutte symbolique visant à hiérarchiser les pratiques culturelles en fonction de leur degré de légitimité. De cette lutte naît une homologie structurale entre position sociale et pratiques culturelles : les membres des classes supérieures ont des comportements culturels légitimes, alors que les membres des classes populaires ont peu accès à ces pratiques. Par conséquent, les systèmes de goût et les pratiques culturelles participent à la reproduction des rapports de domination par l'imposition d'un arbitraire culturel qui correspond à la culture des classes dominantes. Peterson et ses collaborateurs, s'appuyant sur des études autour des goûts musicaux des américains, ont pour objectif de tester le modèle de Bourdieu, en essayant de mettre en relation les goûts musicaux avec la classe sociale des enquêtés. Dans un premier temps, Peterson valide la thèse de Bourdieu, au sens où il admet l'existence d'une hiérarchie culturelle liée au volume et à la structure du capital des individus - le capital culturel étant le capital essentiel au maintien de cette hiérarchie —, mais en même temps, il met en avant une caractéristique des pratiques culturelles des classes dominantes quasi occultée par Bourdieu, à savoir l'hétérogénéité des choix culturels des «dominants», ou leur caractère "omnivore».

2 Toutefois, cette attitude underground s'est vue élargie à presque toutes les formes d'expression musicale contemporaines, donnant lieu à ce que Jean-Marie Seca (2005) appelle l'«underground de masse». 
En considérant ce genre musical comme un bien symbolique ${ }^{3}$, nous chercherons à montrer que, pour cerner au mieux le processus lui octroyant de la valeur - processus préalable à l'établissement d'une hiérarchie —, l'analyse de sa diffusion et de sa réception doit tenir compte des contextes sociaux dans lesquels elle se voit instrumentalisée. Et cela, en fonction d'objectifs mis en œuvre par les acteurs participant à la reproduction symbolique des contextes sociaux : artistes, classes sociales, institutions, acteurs économiques et médias.

C'est donc en nous appuyant sur la notion de contextualisation des formes symboliques telle qu'elle a été développée par J.B Thompson (1990) que nous nous éloignerons de la problématique de la hiérarchisation musicale selon des modalités individuelles de la réception, afin de focaliser sur la fonction sociale des préférences musicales. En décodant les caractéristiques spatio-temporelles du déroulement de l'action, les champs d'interaction sociale - là où priment les ressources individuelles ou propres à un groupe, ainsi qu'un certain nombre de conventions et de règles qui encadrent l'interaction - et, à une échelle plus vaste, les inégalités structurelles entre les différents acteurs, cette approche permet de mettre en relief de possibles rapports sociaux de subordination, de conflit ou de différenciation liés à la réception, mais aussi à la production de cette musique. L'objectif de cette contribution consiste donc à déceler et à analyser ces rapports en nous intéressant principalement aux discours portant sur les caractéristiques objectivables de la musique électronique.

\section{ANALYSER LES CONTEXTES DE PRODUCTION ET DE RÉCEPTION}

Pour Thompson, les diverses caractéristiques des contextes sociaux sont constitutives non seulement de l'action et de l'interaction, mais aussi de la production et des modalités de réception des formes symboliques. Dans ce sens, lorsqu'un individu (ou un groupe d'individus) mobilise des ressources pour produire une forme symbolique spécifique adressée à un ou plusieurs récepteurs, il existe une série de modalités de réception anticipées, prévues ou potentielles - selon la «position» sociale des récepteurs ainsi que d'autres types d'informations détenues à leur égard - , faisant aussi partie des conditions de production.

Par exemple, si un musicien très médiatique et reconnu pour son talent dans un genre musical spécifique change soudainement de style pour attirer un nouveau public, nous pouvons croire qu'il a prévu les modalités de réception de celui-ci, mais aussi présumer qu'il sait qu'il encourt le risque de ne pas plaire à son ancien public. Ainsi, les formes symboliques sont «reçues» par des individus se situant au sein de contextes précis dont les caractéristiques sociales structurent les manières de réception, d'interprétation et de valorisation de ces formes.

Nous conviendrons alors avec Thompson que le processus de réception «n'est pas un processus passif d'assimilation; il est plutôt un processus créatif

3 Les genres musicaux sont considérés ici comme des formes symboliques faisant partie de la culture d'une société. La culture est entendue à la fois dans son sens institutionnel et dans son sens large, faisant référence à toute expression de la vie sociale d'un groupe. 
d'interprétation et de valorisation dans lequel la signification d'une forme symbolique se construit et se reconstruit activement ${ }^{4}$ ». Par le biais de l'interprétation, les modalités de réception des formes symboliques donnent lieu à des processus d'évaluation positive ou négative que l'auteur appelle «processus de valorisation».

\section{LA MUSIQUE ÉLECTRONIQUE, UN GENRE « MINEUR» SOCIALE- MENT VALORISÉ À LIMA}

L’apparition d'une scène de musiques électroniques durant la seconde moitié des années 1990 est le résultat de la forte internationalisation d'un phénomène culturel dont l'établissement à Lima constitue un épisode "provincial» ou périphérique. En effet, il s'agit d'une importation presque à l'identique de formes culturelles trouvant leurs origines dans l'hémisphère nord : l'esthétique et les attitudes mobilisées autour des raves en Europe et aux États-Unis - celles qui évoquent, à partir de films, de vidéo-clips et de romans anglo-saxons 5 , une mouvance culturelle jeune et urbaine des classes moyennes - ont trouvé un public qui s'est identifié à elles. Pourtant, la musique électronique, en tant qu'objet «mondialisé», s'est rapidement engagée dans un dialogue avec les spécificités socioculturelles de la société d'accueil, processus qui a conduit inexorablement à une indigénisation de l'objet nouvellement arrivé (Appadurai 2001). Une fois «transposé» dans la réalité péruvienne, ce genre musical s'est vu circonscrit dans des milieux plus bourgeois, plus restreints et enclins, par souci de distinction, à recevoir des formes culturelles «occidentalisées» et, en même temps, éloignées de celles associées aux classes populaires ${ }^{6}$.

Ainsi, lorsqu'elle arriva à Lima, la musique électronique a été pratiquement confinée au milieu social des jeunes adultes des classes supérieures et plutôt blancs de peau, à la recherche de nouvelles sensations et d'une manière alternative de se divertir7. Cette inscription sociale initiale s'explique non seulement par sa faible diffusion médiatique (elle demeure très peu diffusée à la radio), mais surtout par un certain nombre de facteurs inhérents aux conditions de production et de diffusion de l'expérience festive associée à ce genre musical (Matta 2009). En effet, les fêtes se déroulent aujourd'hui encore dans

4 «The process of reception is not a passive process of assimilation; rather, it is a creative process of interpretation and evaluation in which the meaning of a symbolic form is actively constituted and reconstituted.» (Thompson 1990, p.153).

5 Nous pouvons mentionner, par exemple, certains romans de l'écrivain écossais Irvine Welsh qui décrivent partiellement l'univers des raves dans le Royaume-Uni (Acid House, Trainspotting, Ecstasy), et les films Groove et Trainspotting.

6 Les classes supérieures liméniennes ont toujours essayé d'imiter la consommation culturelle et matérielle européenne. Cette tendance peut être retracée en amont jusqu’à la période coloniale. En effet, depuis leur arrivée sur le nouveau continent, les conquistadors ont d'abord réclamé une alimentation européenne et une fois enrichis, ils ont essayé d'imiter le style de vie des classes supérieures de leurs pays d'origine.

7 Il convient de signaler que les fêtes électroniques ont accueilli en grand nombre des individus qui évitaient les boîtes de nuit diffusant de la musique pour danser en couple (rock, salsa, merengue) pour deux raisons : soit ils n'aimaient pas la musique qui passait dans ces lieux, soit ils n'aimaient pas danser en couple (ou ils n'étaient pas assez doués pour le faire). 
des lieux urbains prestigieux - boîtes de nuit branchées, country clubs, bâtiments historiques réaménagés et résidences privées -, et les billets pour y accéder ont un prix de vente assez élevé par rapport au niveau de vie de la plupart des Liméniens ${ }^{8}$. De plus, l'information sur les fêtes à venir circule sur des supports assez ciblés : le "bouche à oreille» et les réseaux sociaux d'Internet. De ce fait, la scène électronique naissante, tiraillée entre l'imaginaire underground en provenance d'Europe et d'Amérique du Nord et une inscription sociale privilégiée au niveau local, a cultivé un esprit élitiste ${ }^{9}$ qui a produit un fort sentiment d'appartenance et de reconnaissance auprès des premiers participants. Au début des années 2000, les amateurs de cette musique se firent plus nombreux, notamment parmi la jeunesse étudiante des universités privées. Ils commençaient aussi à afficher de façon marquée leur préférence pour les musiques électroniques vis-à-vis d'autres styles considérés comme plus commerciaux ou populaires. La sophistication - le caractère complexe et à la fois novateur perçu par les amateurs dans les compositions musicales -, l'alliance bien dosée entre plaisir hédoniste (exacerbé par l'usage de drogues de synthèse) et maîtrise des codes sociaux, ainsi qu'une mise en scène spectaculaire (décor, son et lumière), ont conforté l'inscription de cette musique dans ce milieu social. Le produit «fête électronique» se rapprochait désormais des soirées «chics» au cours desquelles, en plus de «faire la fête», il était aussi possible de se montrer.

Le succès de ces soirées auprès de cette population s'est vu accompagné d'un processus de légitimation visant à octroyer à cette musique une valeur symbolique et économique sur laquelle elle pourra asseoir les bases de sa pérennité. Les acteurs de la scène électronique (DJs, promoteurs et amateurs) ont ainsi développé une intellectualisation du genre musical, en lui attribuant une position plus élevée dans la hiérarchie culturelle véhiculée par le groupe social qui a le plus contribué à son essor (et le plus apte, économiquement parlant, pour continuer à le faire). Ce mécanisme s'est rendu plus explicite à partir de l'année 2004 : alors que les premiers amateurs évoluaient confortablement à l'intérieur d'une scène de taille restreinte - mais «victime de son succès» -, une population jusqu'alors étrangère à ces manifestations, plus jeune et hétérogène sur le plan socio-économique ${ }^{10}$, adopta cette musique à son tour. Si cet élargissement du public a établi durablement la scène électronique au Pérou - qui, par ailleurs, s'est révélée être une excellente affaire pour les promoteurs —, il a surtout été à l'origine de pratiques de différenciation sociale mises en œuvre par les premiers amateurs vis-à-vis des nouveaux arrivants. L'affirmation du

8 Le prix des grandes soirées avec des DJs internationaux atteint des sommes similaires à celles payées par les clubbers européens, entre 15 et 25 euros.

9 Qui ne s'exprime pas seulement par le prix des entrées aux événements. Un grand nombre de soirées électroniques sont "privées» dans la mesure où seule une poignée de gens est «invitée» à acheter sa place. La gestion de l'accès s'organise sur des réseaux de sociabilité cherchant à préserver un certain degré d'homogénéité sociale au sein des fêtes. On «invite», des amis, des «pairs», des "gens connus», ou des "visages familiers».

10 Cette population est composée par des jeunes issus des classes dites «moyennes émergentes», catégorie composée en grand nombre par des migrants aux origines provinciales et rurales et qui ont vu améliorer progressivement leurs conditions de vie. 
groupe des "pionniers» comme étant la véritable subculture électronique ${ }^{11}$ repose désormais sur des logiques sociales leur permettant de tracer les frontières qui les séparent des "profanes».

Une des modalités par lesquelles s'expriment ces frontières consiste à produire un discours cherchant à légitimer le goût pour la musique électronique en fonction des qualités formelles de celle-ci. Ce discours repose sur deux dimensions valorisantes : l'abstraction et l'évolution. Nous verrons ainsi que cette musique, faite pour le corps «pris» collectivement, "pour la fête de tous avec tous», n'est pas exempte d'un investissement idéologique de la part des acteurs qui la produisent et la consomment.

\section{L'INTELlectualisation d'UNe MUSIQUE SANS PAROLES}

En musique, les paroles - même si elles sont parfois superflues et ont pour principal effet recherché d'accompagner une série rythmique - restent des sources de signification assez importantes et délimitent en quelque sorte le champ de construction d'une composition musicale en matière d'écriture et d'interprétation instrumentale. Dans le contexte latino-américain, par exemple, les paroles sont presque inséparables des musiques de danse (salsa, merengue, entre autres). La plupart du temps, les individus dansent et chantent tout à la fois, ou font semblant de le faire. De ce fait, les musiques avec paroles se trouvent fréquemment tiraillées entre la virtuosité de l'exécution instrumentale et les significations possibles du contenu oral ${ }^{12}$. La musique électronique de danse, elle, a pratiquement éliminé les paroles. Dans les cas où elles sont présentes, leur utilisation dépasse rarement une ou deux phrases (des refrains), souvent répétées en boucle afin de créer un effet sonore; leur intention finale n'étant pas, en règle générale, de faire passer un message. Cette caractéristique octroie à cette musique la possibilité de se voir détachée d'un contexte plus large que les paroles auraient pu aider à construire ${ }^{13}$. Réduite à ses propriétés formelles, la musique électronique est traversée par une démarche de légitimation opérée par ses producteurs et ses amateurs. Elle consiste à mettre en avant le fait que ce genre musical pourrait être jugé exclusivement en fonction de la virtuosité de la composition et de l'exécution - c'est-à-dire dégagé de son contexte festif - , ce qui n'est pas toujours le cas dans la plupart des musiques «chantées», dont les paroles peuvent faire référence à des situations ponctuelles

11 Nous retiendrons ici la definition proposée par David Riesman (1950, p. 361) : «Subculture is a group of people with a culture (whether distinct or hidden) which differentiates them from the larger culture to which they belong». L'auteur opère une distinction entre une majorité qui accepte de manière passive des styles et significations pourvues par le commerce, et une subculture qui cherche activement un style minoritaire en accord avec des valeurs subversives.

12 Ceci est notamment le cas auprès des musiques dites populaires. Pour ce qui est des musiques à contenu oral dites savantes, comme l'opéra, les chorales qui interprètent des compositions de musique classique et, éventuellement, le jazz, le jugement «savant» porte surtout sur les voix, qui sont considérées comme des instruments exécuteurs d’une écriture musicale, et non pas sur les paroles, qui appartiennent plutôt à l'univers dramaturgique. Autrement dit, ce ne sont pas les paroles qui intéressent le plus, mais les sons, les tons, les textures, les qualités instrumentales de la voix.

13 Selon Reguillo (1998), cette absence de message précis confère à cette musique un caractère «global» dans la mesure où elle peut être «comprise» à une échelle transnationale. 
de la vie quotidienne qui font tout leur sens. De ce fait, la musique électronique serait à même de demander au récepteur des "compétences spécifiques " (Bourdieu op.cit. 1979) - mais pas forcément savantes - indispensables à sa compréhension, comme le suggère le DJ péruvien Israel Vich :

Pour s'amuser avec la musique électronique il n'y a pas besoin de compétences, mais pour la comprendre, oui. Par exemple, il faut avoir l'oreille musicale, une aptitude, des connaissances en métrique. Alors, tu peux voir comment le langage musical est plus élaboré et crafted. Ensuite, tu commences à mieux comprendre de quoi il s'agit et pourquoi cette musique te plaît. Mais il ne faut pas arriver au point de devenir snob, comme cela est arrivé avec une musique instrumentale comme le jazz, ou comme cela arrive aujourd'hui avec l'electronica et l'Intelligent Dance Music (styles de musique électronique jugés avant-gardistes) ${ }^{14}$.

Selon Daniel, participant actif de la scène électronique liménienne, les musiques rock et pop des décennies précédentes peuvent aussi constituer un background participant du développement des genres électroniques :

Ce qui se passe maintenant, c'est qu'il y a beaucoup de gens qui veulent être DJ pour devenir cool, pour les filles, mais ils n'ont pas le talent musical. En fait, ils devraient prendre en compte que Christian (Berger), Rodrigo (Lozano), Andy (Dyer) ${ }^{15}$, ceux qui mixent en ce moment, connaissent aussi d'autres styles. Ils ont écouté d'autres musiques auparavant, ils ont un background qui fait que les lignes mélodiques, les lignes fortes d'autres styles ressortent au cours de leur prestation, par exemple, la musique des années 1980, la musique des années 1970, Led Zeppelin, tout cela influence leur façon de mixer. Aujourd'hui, il y a des jeunes gens intéressés par la mode autour des DJ, et qui n'ont écouté que de la musique électronique ${ }^{16}$.

DJ Andrés Dyer va un peu plus loin. Pour lui, "comprendre» la musique électronique n'implique pas seulement d'avoir l'«oreille musicale» : selon lui, un individu ayant une vaste culture générale ou ayant reçu une «bonne éducation» serait plus apte à assimiler et à apprécier son travail derrière les platines et les synthétiseurs. À en croire ses propos, cela concernerait une partie très minoritaire de la population :

Ce qui arrive au Pérou, c'est que les gens ne sont pas très cultivés, disons qu'ils n'ont pas beaucoup de connaissances, ils n'ont pas une grande amplitude (mentale). Donc, ce sont les gens appartenant aux secteurs A et $\mathrm{B}^{17}$ qui, en principe, ont plus d'éducation et, en conséquence, plus de culture apprécient le plus cette musique. Et je crois que c'est là le noud de la question. Par exemple, si tu fais écouter la musique de Richie Hawtin

14 Entretien avec l'auteur, le 16 septembre 2008 à Lima.

15 Trois DJ parmi les plus en vue de la scène électronique liménienne.

16 Entretien avec l'auteur, le 5 novembre 2007 à Lima.

17 La classification socio-économique de la population péruvienne se divise en 5 grands niveaux juxtaposés verticalement, sous forme de pyramide, de $\mathrm{A}$ à $\mathrm{E}$ (de haut en bas) : A (classe supérieure), B (classe moyenne-supérieure), C (classe moyenne-inférieure), D (classe inférieure ou "populaire») et $\mathrm{E}$ (pauvreté). 
(DJ et producteur canadien, précurseur de la minimal techno) à quelqu'un qui n'a même pas écouté du rock durant toute sa vie et, ensuite, tu la fais écouter à quelqu'un qui n'a jamais écouté de la musique électronique, mais qui connaît bien d'autres styles, tu noteras des différences. Si cette personne a eu la chance d'avoir une bonne éducation, si elle a voyagé et a connu des styles différents, cette personne ne dira pas c'est la musique du démon! Pardon, je rigole. Mais, en revanche, si cette personne n'a pas eu cette chance, elle dira : qu'est-ce que c'est bizarre comme musique! [...] Je suis sûr que quelqu'un qui n'a pas de notions musicales et qui ne connaît pas la musique électronique aura des problèmes pour comprendre, pour assimiler ce que je fais. Alors, mon objectif en tant que DJ est que ça ne lui déplaise pas. Je ne fais pas non plus des choses super recherchées ou super expérimentales, mais ça reste quand même assez difficile à comprendre pour quelqu'un qui ne connaît pas.

Ces propos révèlent qu'il existe dans la musique électronique une démarche de légitimation faisant appel à un bagage culturel plus important comme condition sine qua non pour « apprécier» cette musique. Plus encore, lorsque DJ Andrés Dyer précise que la minimal techno de Richie Hawtin ne pourrait pas être appréciée par quelqu'un qui n'a jamais écouté du rock (musique urbaine par excellence dans les pays de la région andine), il établit un lien de causalité entre les origines sociales de l'individu en question - résolument provinciales et modestes si l'on se tient à l'évaluation de notre informateur - et son manque d'aptitude à interpréter une musique électronique plutôt cataloguée comme «cérébrale». Pour reprendre cette idée dans les termes de Hans Robert Jauss (1972 [1990]), le corpus musical intériorisé par cet individu ne concèderait ici aucune référence implicite ou quelque élément de reconnaissance du sens de l'oeuvre qui puisse orienter la réception de la musique de Hawtin. Il s'agit donc d'un décalage qui ne produirait aucune valeur esthétique chez l'auditeur ${ }^{18}$.

Pour leur part, DJ Israel Vich et Denis confirment l'effacement des signifiants oraux, conférant ainsi à cette musique une aura abstraite qui laisse plus de place à des interprétations relatives à son processus de construction, en particulier lorsqu'ils emploient des termes comme «recherché», « expérimental», «langage élaboré », "crafted» et «lignes mélodiques».

Ce constat nous permet d'opérer un rapprochement entre ce discours et celui développé autour de l'art abstrait ${ }^{19}$, considéré assez souvent comme une expression culturelle de réception difficile et à la portée d'un public réduit ${ }^{20}$.

18 Si nous la reprenons en utilisant la terminologie d'Umberto Eco (1992), la transaction entre la compétence de l'auditeur (la connaissance du monde partagée par l'auditeur) et le type de compétence que la musique de Hawtin postule pour être appréciée serait trop difficile, voire improbable.

19 D'ailleurs, DJ Israel Vich considère qu'une bonne partie de la musique électronique, celle qu'il considère comme étant à l'avant-garde (Intelligent Dance Music et minimal techno, par exemple), commence à se débarrasser de la prédominance «dansante» pour devenir plus «conceptuelle et souvent chaotique» (entretien op.cit.)

20 L'enquête sur la consommation d'art abstrait parmi l'upper class et la working class de la région new-yorkaise, réalisée par le sociologue nord-américain David Halle (1992), montre que ce type de "haute culture» attire, en effet, l'intérêt d'une minorité. Cependant, son étude montre aussi que l'importance des compétences culturelles dans la réception ou la «compréhension» de l'œuvre d'art est nuancée : la perception des enquêtés - des propriétaires d'œuvres d'art abstrait - bascule notam- 
Pourtant, nous devons admettre que, bien que le développement du goût musical puisse demander du temps et des notions difficiles à obtenir, l'étalage des préférences musicales n'a pas forcément, de nos jours, un effet déterminant dans la démarcation sociale, c'est-à-dire qu'il ne constitue pas une barrière insurmontable entre groupes sociaux. Comme le remarque Joel Chadabe (2002), les avancées technologiques rendent plus démocratique l'art électronique et donnent à un public dépourvu de connaissances ou de compétences musicales spécifiques la possibilité d'interagir de façon créative et complexe avec un processus musical $^{21}$. Toutefois, derrière cette apparente démocratisation se dresse la véritable nature de la technologie, faite d'inventions et de réinventions infinies et d'un développement souvent exprimé en termes d' «évolution», concept laissant place à la différenciation.

\section{LA TECHNOLOGIE COMME SUPPORT DU DISCOURS LÉGITIME : ÉVOLUTION VS. "INVOLUTION"}

La musique électronique dépend d'un ensemble de supports (synthétiseurs, logiciels, etc.) de plus en plus diversifiés et d'utilisation facile pour quelqu'un de familiarisé avec les nouvelles technologies. Conçus notamment pour travailler les sons, ces supports permettent de nombreuses possibilités de composition en élargissant le spectre sonore et rythmique. Le compositeur de musique électronique trouve alors des marges d'agencement plus grandes concernant le devenir de son œuvre et de son statut d'artiste. Il a notamment le pouvoir de choisir son niveau de rapprochement au marché (être très connu dans des milieux plus larges ou bien utiliser de multiples hétéronymes pour rester dans le milieu underground), faire circuler son œuvre sur divers supports et circuits, ou bien privilégier un nombre réduit d'entre eux. Il peut aussi évoluer dans différents styles électroniques selon ses priorités individuelles.

Étant donné que les machines offrent la possibilité d'élargir l'éventail de la «sophistication», il est important d'observer comment se situent les genres musicaux par rapport à cet éventail. Ceci devrait amener à repérer de nouvelles hiérarchies entre les genres qui se rapprochent du «moins sophistiqué», et ceux qui se rapprochent du "plus sophistiqué». Cette hiérarchie peut être établie au sein d'un même style ou entre différents styles. Ainsi, pour Ben Neill, la musique électronique contiendrait ses propres exemples de «haute culture» et de "culture populaire»; la différence centrale entre ces deux catégories résiderait dans le concept de rythme. Autrement dit, c'est le rythme qui tracerait la ligne de division «entre le sérieux et le moins sérieux, le viscéral et l'intellectuel» (Neill 2002, p. 3). De ce fait, et malgré l'utilisation de différents outils conceptuels et matériels dans la composition musicale, l'approche

ment entre leur goût pour le design (décoratif) et le fait qu'elle permet à l'imagination de «s'envoler» (subjectivité individuelle), et ceci pour l'ensemble des groupes sociaux étudiés. En revanche, les raisons évoquant une culture artistique "abstraite» sont considérablement minoritaires. Ceci indique que certaines formes de la "haute culture» ne nécessitent pas de compétences spécifiques pour être consommées et appréciées.

${ }^{21}$ Reste à savoir, comme le souligne Timothy Taylor (2001), de quelle manière cette technologie se démocratise dans un contexte spécifique et, surtout, pour qui elle devient démocratique. 
esthétique différenciant la «haute musique électronique» de la «musique électronique populaire» peut être mieux définie par la présence ou l'absence de rythmes répétitifs (repetitive beats), les styles plus "sérieux" étant ceux qui comportent le moins de répétitions. Pourtant, si la musique électronique de danse est, grâce à la répétition, caractérisée comme «physique», tandis que les styles plus «pointus» recherchent la complexité et la dissonance, le travail de certains compositeurs et performeurs contemporains remet progressivement en question cet écart ${ }^{22}$.

La relation entre ce genre musical et le développement technologique entraîne alors de nouvelles façons de penser l'art et, par conséquent, la possibilité de développer des discours de légitimation susceptibles d'ériger des barrières entre les nouvelles expressions artistiques. Elle peut, de ce fait, favoriser des interprétations attribuant une plus grande valeur aux expressions le plus en phase avec les nouvelles découvertes ${ }^{23}$ ou avec l'utilisation expérimentale de la technologie, notamment à travers la différenciation et l'opposition entre ce qui est «commercial» (une musique perçue comme étant plus «digérable» et «populaire») et ce qui est underground (une musique alors plus axée sur la recherche et le constant dépassement des compétences des musiciens). Par exemple, le passage du style trance $e^{24}$ - qui était celui qui prédominait lors des débuts de la scène électronique liménienne - vers différents styles de house (deep ${ }^{25}$ ou progressive ${ }^{26}$ ) - styles en vogue depuis quelques années - est saisi par certains consommateurs et producteurs non pas comme un changement dans

22 Par exemple, l'œuvre des Anglais Aphex Twin (Richard D. James) et Michael Paradinas, de l'Américain Sutekh (Seth Horvitz) et des Canadiens Richie Hawtin et Akufen (Marc Leclair), pour n'en citer que quelques-uns, prouve que les compositeurs de musique électronique sont désormais capables de travailler autant avec les processus sonores les plus abstraits et les techniques les plus expérimentales qu'avec des séquences rythmiques faisant référence à des styles musicaux de danse préexistants.

23 A ce sujet, Taylor (op.cit. 2001, p.8) rappelle la place centrale qu'occupe la technologie dans l'idéologie occidentale, fondée sur le progrès et l'avancement de la science : «... by the middle of the eighteenth century one of the most important and sometimes the most important way that Europeans judged other cultures was by their scientific and technological advancement.» (... vers la moitié du dix-huitième siècle, une des façons les plus courantes et, souvent, la plus courante utilisée par les Européens pour juger les autres cultures était de mesurer leur avancement scientifique et technologique [traduction personnelle]).

24 Style apparu en Allemagne vers le début des années 1990, postérieurement à la techno et à la house. Il se caractérise par une recherche systématique de lignes mélodiques répétitives et planantes obtenues par l'utilisation de filtres dont les fréquences de coupure varient dans le temps. L'«esprit» de cette musique vient du fait que la musique et la danse peuvent altérer la perception sensorielle de l'auditeur et le transporter dans un état d'extase hypnotique et méditative, d'où son nom. Le tempo d'un track de trance se situe entre 130 et 170 BPM (Beats Per Minute).

25 Version plus underground de la house qui fusionne des éléments de la house de Chicago, du soul des années 80 , du jazz-funk et de la techno de Detroit. Le qualificatif deep renvoie à la dimension introspective de ce style, proche du jazz et de la soul, alors que la house classique, plus "gaie», est davantage liée aux racines disco et funk. Le tempo de ce style se situe entre 125 et 135 BPM.

${ }^{26}$ Le terme progressive fait référence à une construction graduelle où l'énergie arrive vers le récepteur de façon progressive, soit au cours d'un track, soit au cours d'un même set ou album. La progressive house puise ses origines en Grande-Bretagne durant les années 1990. Le son électronique progressif consiste typiquement en un rythme house - sans les vocaux - avec des lignes de basses profondes et planantes et un déploiement progressif créant une atmosphère mélancolique. Le tempo de ce style se situe autour de 135 BPM. 
les tendances ou modes musicales, mais comme une évolution. Les propos de deux de mes informateurs illustrent cette idée :

Quand tu écoutes longtemps un certain style musical, tu commences à évoluer. C’est comme chez tout être humain : le temps passe et tu commences à mûrir, les attitudes de quand tu étais plus jeune commencent à changer, tu commences à les écarter [...] Il se passe la même chose en matière musicale, tu fais mûrir ton ouïe, tu affines ton goût ou bien, si tu ne l'affines pas, tu désagrèges les autres styles par saturation musicale ${ }^{27}$.

Tout évolue, la musique aussi. [...] Je ne crois pas que les styles électroniques plus anciens aient perdu de la valeur, mais c'était de la bonne musique pour une autre époque. Mais ce qui est vrai, c'est qu'à Lima beaucoup de gens se positionnent en fonction de la musique que fait le DJ, et les DJs commerciaux peuvent ne pas être bien vus. (DJ Andrés Dyer, entretien op.cit.)

Ainsi, un style dit commercial est, dans ce cas, considéré comme "peu évolué», tandis qu'un style de diffusion plus restreinte est perçu comme plus "évolué», plus "complexe», ou reconnu comme étant de la «meilleure musi$q^{2} e^{28} »$. En prenant comme point de départ ce constat, certains amateurs se positionnent et se distinguent par rapport à ce registre axé sur la rareté et l'expérimentation musicale avec des machines. Cependant, la conceptualisation de «l'évolution» dans les arts ne saurait s'expliquer par une «évolution du goût» donnant lieu à un changement dans les préférences (en effet, il n'y a pas « un goût» qui évolue; il change simplement), mais par une transformation dans les catégories de réception (Heinich 1998). L'idée d'évolution doit donc être comprise en fonction des modalités du "regard» susceptibles d'apparaître, de disparaître ou de se superposer à d'autres en fonction des intérêts, des compétences et des stratégies des acteurs en interaction. D’après les propos qui viennent d'être présentés, nous avons pu établir une relation entre le «discours évolutif» et une dynamique de différenciation sociale engendrée par ceux qui se sentent aptes à tirer profit de la technologie (à la «comprendre» et à se l'approprier) vis-à-vis de ceux qu' ils considèrent, à cause de leur manque de capital culturel et/ou économique ${ }^{29}$, contraints ou limités à une attitude de réception moins élaborée et valorisante. Mais, aussi, ce discours peut être utilisé pour établir des jugements de valeur au sein du même groupe et renforcer la posture hégémonique de façon arbitraire :

Les gens aiment bien dire : «moi j'écoute de la meilleure musique que toi ». Cela arrive tout le temps, parce que ça te donne un air de supériorité

27 Gustavo, promoteur de fêtes électroniques et directeur d'une école de DJ. Entretien avec l'auteur, le 17 août 2007 dans les bureaux du «DJ College».

${ }^{28}$ La récupération de la musique par l'industrie culturelle de masse a contribué à ce que certains genres musicaux dits "commerciaux" soient considérés comme étant peu évolués, formatés et ciblés pour un public très large qui cherche principalement à se distraire (inattention), à échapper à des obligations quotidiennes. À ce sujet, nous pouvons citer les écrits de Theodor W. Adorno (1941) et une critique à l'égard de la position ethnocentriste du philosophe allemand (Williams 2005).

29 S'exprimant souvent dans le discours comme des manques en «éducation». 
par rapport à l'autre. Auparavant, nous critiquions les gens qui écoutaient Tiësto (DJ hollandais, figure emblématique pour les Liméniens du DJ superstar et «commercial»), mais il faut respecter leur choix [...] Moi, par exemple, j'ai un ami qui continue à écouter de la trance et, avant, on l'embêtait. Nous lui disions : «toi, tu n'as pas évolué», mais si tu te rends compte, huit ou dix ans sont passés, et s'il continue à écouter cette musique c'est parce qu'il l'aime vraiment, ce n'est pas parce qu'il n'a pas évolué, mais parce qu'il a développé un autre goût musical. Si moi j'étais DJ, je passerais un autre style de musique, pas celui-là. Si lui devenait DJ, il passerait certainement un style différent. Il s'agit de différents goûts musicaux qu'il faut respecter. Mais, ici, les gens ne respectent pas. (Daniel, entretien op.cit.)

Cette instrumentalisation du discours sur l'évolution laisse place à une réflexion autour du concept d'involution (en anglais), développé par l'anthropologue américain Alexander Goldenweiser (1936) pour décrire des formes culturelles (comme le tatouage maori ou l'architecture gothique) qui, ayant atteint une forme définitive et reconnaissable, continuent à se développer en devenant plus complexes à l'intérieur d'elles-mêmes. L'involution ne doit pas être entendue comme une régression («involution», en français), mais comme une mutation qui se trouve fortement inscrite dans des patterns ${ }^{30}$ bien déterminés jouant un rôle d'encadrement au sein d'une société. Le pattern vient ainsi former une sorte de halo qui, par la transmission qui en est assurée, encadre les innovations. Bien qu'il écarte ou limite l'utilisation de certaines formes symboliques, il n'empêche pas pour autant de jouer avec celles qui sont permises. Ainsi, plus les limitations déterminées par le pattern sont fortes, plus les changements seront issus de réélaborations pouvant conduire à une complexité extrême en apparence, ce qui donne lieu «à une variété dans l'uniformité, à une virtuosité dans la monotonie» (Goldenweiser 1936, p. 103). L'involution fait donc référence à une évolution dans (in) une structure définie.

Ce concept s'avère d'une grande utilité pour comprendre la réception musicale tout en montrant que la "complexité» - notion à l'origine du processus de distinction selon le barème de la sophistication - est relative, souvent trompeuse, et pouvant être construite avec un nombre réduit d'éléments. Par exemple, Goldenweiser remarque dans les «fugues» de J.S Bach une limitation délibérée de l'inventivité concernant les éléments mélodiques. Cependant, le développement et l'inventivité sont toujours présents, mais ils prennent forme à partir d'un jeu sur la base mélodique fondé sur la répétition de multiples combinaisons et recombinaisons. Le résultat de ce processus est une structure musicale plus complexe, capable de «tromper l'oreille».

L'application de la théorie de l'involution trouve alors sa pertinence dans la composition de musique électronique, celle-ci étant essentiellement construite

30 Le pattern, que nous pouvons définir comme un modèle ou un schéma de pensée inscrit par l'action de la société dans l'individu, permet de confirmer le processus de développement d'une culture et, à la fois, pose ses limites. Dans les sociétés dites traditionnelles, la force du pattern est indiscutable. Elle est mise à l'épreuve et confirmée par le biais des rites et des rituels, souvent associés à des formes de religiosité (Benedict 1934 [2006]). 
autour de combinaisons et recombinaisons de plages sonores. L'involution pourrait même expliquer l'estime que les amateurs liméniens affichent pour ce style musical, contrairement à d'autres perçus comme moins sophistiqués, moins «travaillés», et donc moins prestigieux.

\section{Conclusion}

Situer le contexte de développement de la musique électronique à Lima, l'espace social de sa diffusion, la condition sociale de ses amateurs et un certain nombre de conventions partagées par ses producteurs, nous a permis de dégager un certain nombre d'aspects permettant d'approcher autrement la fonction sociale de la musique et la question de la hiérarchisation culturelle. Nous avons vu que le succès de la musique électronique et de ses manifestations auprès d'une minorité de Liméniens (une fraction de jeunes adultes des classes supérieures) est redevable d'un discours qui attribue à celle-ci une valeur artistique supérieure par rapport à d'autres expressions musicales «non savantes» et dansantes. Pourtant, sa position élevée concerne principalement la hiérarchie culturelle détenue par un seul groupe social. Ainsi, les amateurs participent d'une scène où l'entre-soi est assez fort, tout en justifiant un «avantage culturel» par rapport à tout ce qui peut être considéré non seulement comme étant «à la traîne», "simple», ou "peu évolué» en termes musicaux (processus de valorisation symbolique), mais encore en termes sociaux. Cela montre bien que le capital culturel mis en exergue dans le discours des pionniers de la scène électronique peut être facilement "personnifié» (embodied). Par ailleurs, cette dynamique se conforte dans le discours et les stratégies des agents de production (DJs, promoteurs d'événements, etc.). Chargés de perpétuer la scène électronique le plus longtemps possible, ils essaient de reproduire cet "avantage» tout en en retirant le plus de bénéfices possible (au niveau économique comme au niveau symbolique). Ce constat nous rapproche en quelque sorte de l'utilisation du concept de «subculture» de Bourdieu (op. cit. 1979) par Sarah Thornton (1995) dans sa description de la club culture au Royaume-Uni. Dans ses conclusions, l'auteur mobilise l'idée que chez les clubbers et les ravers, groupes partageant un même intérêt pour certaines pratiques (légales ou illégales), un même goût musical et un fort sentiment d'appartenance, les effets de hiérarchie sociale sont toutefois présents en raison d'une répartition inégale de "capital subculturel» : connaissances spécifiques, comportements, styles... en somme tout élément pouvant montrer aux autres que l'on est quelqu'un de bien informé ("being in the know»).

Mais si Thornton observe, dans cette forme de capital, une attitude rebelle face au joug parental et aux déterminismes de classe, le discours des amateurs et producteurs de musique électronique à Lima renvoie davantage à la vision hiérarchique de la société véhiculée par les groupes dominants et à une utilisation du capital culturel propre à ces groupes dans une stratégie de reproduction sociale. 


\section{RÉFÉRENCES}

Adorno, Theodor W et George Simpson. 1941. «On Popular Music». Studies in Philosophy and Social Science 9:17-48.

Appadurai, Arjun. 2001. Après le colonialisme. Les conséquences culturelles de la globalisation. Paris : Payot.

Benedict, Ruth. 1934 [2006]. Patterns of Culture. Boston : Mariner Books.

Bourdieu, Pierre. 1979. La distinction : critique sociale du jugement. Paris : Minuit.

Chadabe, Joël. 2002. «Remarks on Computer Music Culture». Computer Music Journal 24, n $4: 9-11$.

Coulangeon, Phillipe. 2003. "La stratification sociale des goûts musicaux». Revue française de sociologie $44, \mathrm{n}^{\circ} 1: 3-33$.

Donnat, Olivier (dir.). 2003. Regards croisés sur les pratiques culturelles. Paris : La Documentation française.

Eco, Umberto. 1992. Les limites de l'interprétation. Paris : Livre de Poche.

Goldenweiser, Alexander. 1936. «Loose Ends of Theory on the Individual Pattern, and Involution in Primitive Society». Essays in Anthropology. 99-104. New York : Books for Library Press.

Halle, David. 1992. "The Audience of Abstract Art: Class, Culture, and Power». Cultivating Differences, sous la dir. Michèle Lamont et Marcel Fournier, 131-151. Chicago : The University of Chicago Press.

Heinich, Nathalie. 1998. Le triple jeu de l'art contemporain. Paris : Minuit.

Jauss, Hans Robert. 1972 [1990]. Pour une esthétique de la réception. Paris : Gallimard.

Matta, Raúl. 2009. Enjeux sociaux d'une consommation "haut de gamme». Étude sur les logiques marchandes et sociales au cour de deux expressions culturelles dans la ville de Lima : l'expérience gastronomique et les fêtes de musique électronique. Thèse de doctorat en sociologie, Université Paris 3Sorbonne Nouvelle.

Neill, Ben. 2002. "Pleasure Beats: Rhythm and the Aesthetics of Current Electronic Music». Leonardo Music Journal 12:3-6.

Peterson, Richard et Albert Simkus. 1992. "How Musical Tastes Mark Occupational Status Groups». Cultivating Differences, sous la dir. Michèle Lamont et Marcel Fournier, 152-168. Chicago : University of Chicago Press.

Reguillo, Rossana. 1998. «El año dos mil, ética, politica y estéticas : imaginarios, adscripciones y practicas juveniles. Caso Mexicano ». Viviendo a toda, sous la dir. de Mario Margulis et al., 57-82. Bogota : Siglo del Hombre Editores.

Riesman, David. 1950. "Listening to popular music». American Quarterly 2 : 359-371.

Seca Jean-Marie. 2005. "Formatage du ressentir et représentations underground». Sociétés $90:$ 57-69.

Taylor, Timothy. 2001. Strange Sounds. Music, Technology and Culture. Londres : Routledge.

Thompson, John B. 1990. Ideology and Modern Culture. Stanford : Stanford University Press. 
Thornton, Sarah. 1995. Club cultures: Music, Media, and Subcultural Capital. Connecticut : Wesleyan University Press.

Williams, Patrick. 2005. «Le déni d'Adorno ». L’Homme 175-176, nº3-4 : 419-425.

\title{
RÉSUMÉ
}

La musique ne saurait être analysée per se, car elle est enracinée dans les contextes sociaux dans lesquels elle se produit et se développe. En analysant les caractéristiques de l'espace social de diffusion de la musique électronique dans la ville de Lima, nous serons en mesure de comprendre la façon dont ce genre musical «mineur» est aujourd'hui devenu un vecteur de distinction sociale et d'élitisme.

\begin{abstract}
Music cannot be analyzed per se because it is thoroughly embedded in the social contexts in which it is produced and developed. By analyzing the characteristics of the social space in which electronic dance music is diffused in Lima, we will be able to understand how this "minor" music genre became an instrument of social distinction and elitism.
\end{abstract}

